\title{
Nanotechnology Through Lesson Plans in the 7th Grade of Primary School in Croatia
}

\author{
Marina Molnar \\ Primary School Stobreč, Stobreč, Croatia
}

\begin{abstract}
An idea came to the author when she was on a professional conference named "Nanotechnology: Digiskills_-Digital Competence in Education (Inverted Classroom)," which awakened the author's curiosity of how much her students know about nanotechnology, especially about the term "nano" and photography. The plan was to revise: 1 . What is "pixel" and what is the difference between photography and digital photos? 2. Is there any connection between nanotechnology and photography? 3. How much nanotechnology has an effect on quality of photos and is there any effect at all? and 4 . Whether photos can be protected with assistance of nanotechnology. The students were not excited about exploring connections between nanotechnology and photography/digital photography, but were interested in enquiring nanotechnology in the field of medicine, cosmetics, environmental protection, and robotics. The students discussed about the meaning of the term "nano," and linked it with the curricula of mathematics, physics, biology, and chemistry. They explored areas in which nanotechnology can be developed as well as various nanomaterials that exist. They were interested in finding answers to the question of how nanomaterials act in medicine, cosmetics, and environmental protection, as well as researching "nanobots"-Are they our future or present? They explored how nanomaterials act on the environment, or if they have had an impact on our health or health of our environment. After they collected materials, the students formed them into Websites, mobile applications, online presentation, and glogs.
\end{abstract}

Keywords: nano, nanotechnology, nanobots, nano medicine, nano cosmetics, Websites, mobile applications, interactive posters/glogs, online presentations

\section{Introduction}

In primary schools, it is often seen that through various school subjects permeating common burning issues, such as environmental protection or addictions, students soon became bored because such topics are mostly repeated. This work arose out of the author's curiosity about how students react to completely new or less familiar topics, and are now using new and innovative teaching methods. In addition, it aims to show how to solve information technology (IT) problems in innovatory ways and to find solutions in one of the new online platforms and programs.

Prefix "nano" comes from the Greek word "nannos" which means "dwarf," and nanoparticles are smaller than cells in the human body. "Nanotechnology is science, engineering, and technology conducted at the nanoscale, which is about 1 to 100 nanometers" (National Nanotechnology Initiative, n.d.). It has the ability to

\footnotetext{
Marina Molnar, master of primary education and informatics, teacher of IT, Primary School Stobreč.

${ }^{1} \mathrm{http}: / /$ www.pip.hr/dld/premaz_letak_004.pdf.
} 
manage materials in nano size in order to create structures that have new properties due to their size, shape, and composition. Nanoparticles are more reactive to some other molecules which are a result of their larger surface area per weight then other greater particles (UnderstandingNano.com, n.d.). Also, nanoparticles are used in various areas of people's daily lives, such as environment, health care, textiles, industrial, renewable energy, food, electronics, etc. (Czwyk Marić, 2014b).

The subject of nanotechnology is very interesting, because it is a less known and infrequent topic, especially among students of primary schools. In this paper, the main goal is to check the effect of new themes throughout the lesson plans and how students are accepting various ways of teaching and learning and keeping them motivated to complete all predicted tasks. Also, it aims to improve students' knowledge to a new level by experiential learning.

\section{Basic Project Information}

The projects target group was 12 students of 7th "A" Grade of Primary School Stobreč who attended informatics. The students are at the age of 13-14. The implementation starts on April 30 until June 11, 2015, through lesson plans envisaged by the end of the school year. The project is connected with curricula of math, physics, biology, and chemistry, and with the curriculum of Primary School Stobreč named "Health, Safety, and Environmental Protection." Technical requirements that are used in the implementation are personal computers, projector, mobile phones, Internet, video player, Web tools (Glogster), Web services (Edmodo and e-mail), Web-hosting services (Weebly and IBuildApp), and an online software for creating presentation (Prezi).

Teaching units were conducted:

(a) On April 30, 2015-Revision of the lesson: Web development - 59-60 hours;

(b) On May 12, 2015-The structure of the Internet (processing of teaching material and exercise) - 61-62 teaching hours;

(c) On May 14, 2015-Other Internet services (processing of teaching material and exercise) -63-64 teaching hours;

(d) On May 21, 2015-Other Internet services (exercise and repetition) - 65-66 teaching hours;

(e) On May 28, 2015-Internet service providers (processing of teaching material) and connect to the Internet (processing of teaching material) - 67-68 teaching hours;

(f) On June 11, 2015-Revision of the lesson: Web development and Internet; Justification achievements and concluding scores $-69-70$ teaching hours.

\section{Keywords of the Curriculum for Primary Schools}

Keywords of the curriculum for primary schools include (Ministry of Science, Education and Sports, Republic Croatia, 2006):

(a) Acquaint interface, text, and background, and insert graphic elements;

(b) Index page, the text as a link, and the image as a link;

(c) Graphics and backgrounds, simple tables, and formatted text;

(d) The structure of a Hypertext Markup Language (HTML) document, HTML document tags (tag), and simple HTML page description;

(e) Tag P, tag A, and tag IMG;

(f) Planning the structure of the page management structure, compilation, and publication of the page; 
(g) Server, client, and communication methods;

(h) Remote file transfer (FTP);

(i) Internet access and hosting of Websites;

(j) Types of modems and modem role.

\section{Educational Achievements of the Curriculum for Primary Schools}

Educational achievements of the curriculum for primary schools are:

1. Making a simple personal Website;

2. Inserting a picture element in Website and turning it into a link to another page;

3. Using the table as an element of the Webpage;

4. Interpreting simple HTML page description;

5. Being able to add parameters to the basic tag;

6. Formulating the structure of the page;

7. Being able to explain the methods of communication within the Internet;

8. Describing the described Internet services;

9. Explaining the role of the server;

10. Explaining the need for faster Internet connections in the future.

\section{Learning Outcomes}

Students will be able to:

1. Appoint the initial and most important page on the Website;

2. Describe what a Webpage is;

3. Identify and know the meaning of the abbreviation of HTML;

4. Compare as graphic (HTML) editors;

5. Define acronym WYSIWYG;

6. Describe code with which we can connect the two sites;

7. Determine what kind of image format to use when creating a Webpage;

8. Describe the tag used to display the image in an HTML document;

9. Describe tags for the title; the letters that are bold, underlined, and italicized;

10. Become conscious of how to create a new HTML document;

11. Describe which command creates a new link;

12. Distinguish between tags $<$ html $>$, $<$ head $>$, $<$ title $>$, and $<$ body $>$;

13. Explain why the tags have their final tag;

14. Explain the elements that make up the table;

15. Identify the structure of the Internet and the ways of communicating with it;

16. Explain the structure of the Internet and the role of the server;

17. Evaluate the quality of the content and process know contents from the Internet;

18. Explain the modes of the communication within the Internet;

19. Explain the protocols for communication;

20. Describe what World Wide Web (WWW) is;

21. Explain the structure of the Internet and terms the client and the server;

22. Explain the role of the server and list server types;

23. Itemize and describe the various services of the Internet (forums, FTP, chat, e-learning, and e-mail); 
24. Distinguish the tools of Web 2.0;

25. Distinguish between social networks and their advantages and disadvantages;

26. Develop their own interactive posters (Glogster);

27. Develop a Webpage using a Website builder Weebly;

28. Develop mobile applications using a Web platform IBuildApp;

29. Create an account on social networks for students (Edmodo) and use it in classroom or home;

30. Use one of the basic services of the Internet-E-mail;

31. Use a tool for sharing videos-YouTube - students can find videos of nanotechnology and nanomaterials that are appropriate, interesting, and understandable to them;

32. Publish their site on the Internet;

33. Explain the meaning of the FTP service;

34. Explain the role of the modem;

35. Explain who are service providers (ISP);

36. Explain the hosting of Webpage;

37. Explain what is necessary for creating an account (username, password, and e-mail address);

38. Explain how to connect to the Internet;

39. Explain the term of "nano," nanotechnology, nanomaterials, and nano computers;

40. Explain in what area nanotechnology can be found;

41. Explain the term "bit" and contradistinguish with "nano bits";

42. Comparing "nano" and "bits";

43. Relate nanotechnology with other subjects: math, chemistry, physics, biology, and everyday life;

44. Explain what the "nanobots" are;

45. Explain the role of nanomaterials in medicine, cosmetics, and in nature conversation and environmental protection;

46. Use and develop an English language in the classroom and apply it in everyday life.

\section{Activities and Innovative Solutions of Resolving Informatics Problems (Context of Practice)}

\section{Revision of Lesson: Web Development}

Students have discussed about the new terms of "nano" and "nanotechnology," and expressed their thoughts and ideas about the meaning of them. Watching the video "What is Nano?", ${ }^{2}$ they draw their conclusions of nano, of any materials nano is made of, how small is nano, and is it visible to the human eye, and their opinions of whether nanotechnology is our future or present already. Also, they used and developed their English language in the classroom, as well as helped each other master the English language.

The author and the students have revised the lesson "Web Development" on the way they did not revise yet, through creating a Website with assistance of Website builder called Weebly. ${ }^{3}$ It is important that the students can describe the known material: HTML is the language that describes the appearance of the Website; WYSIWYG means What You See Is what You Get, so that lessons that have been learned can be applied in learning new material and in everyday life. The students discussed that "WWW" signifies "World Wide Web"

\footnotetext{
${ }^{2}$ YouTube video: https://www.youtube.com/watch?v=Dxq-ffp3jqA.

3 http://www.weebly.com/.
} 
and it is a multimedia information service of the Internet. For start, the author and the students created an account on Weebly, where they can login their accounts by e-mail addresses and passwords. Weebly offers them a possibility of creating Websites, blogs, or online stores. The students pick up production site custom theme, which best fits to nanotechnology. In addition, it is good to be cautious when choosing themes, because different topics require appropriate theme. Among other things, one of the main objectives of the lesson is to link concepts of the major page of the Website, terms, such as "tag" and tag for begging and the end of Webpage. $^{4}$ On the left side is a toolbox with elements of the Webpage. The first tab, on the top of the Web builder, is called "Build" with all its elements. The second tab, "Design," helps to pick up custom themes, font types, adding social networks, possibility for amending design of Webpage with HTML/CSS code, etc.. The students have explained code for bold, italic, and underline letters, ${ }^{5}$ and font types and colours, ${ }^{6}$ as well as code for background colors ${ }^{7}$ and background image. ${ }^{8}$ Third, tab "Pages," marks pages within Webpage, which are their integral part and have compared it in which way is possible to create new page inside Webpage in Notepad/KompoZer or Notepad++. ${ }^{9}$ The element, in which pictures are inserted in Weebly, associated with the HTML code, for example, <img src="picture.jpg" $>$ or $<$ img $s r c=$ "picture.gif" $>$. The students were arguing about the benefit of tags $<$ head $>,</$ head $>,<$ title $>,<$ title $>,<$ body $>$, and $</$ body $>$, which is important for deeply understanding of the elements of the Websites and the code behind them. In Weebly, the title will be showed in the part which is called "My site" and it will be visible on Web browser title bar. Weebly enables inserting logo, pictures or pictures galleries, audio and video, maps with locations, documents, other pages on Internet, and so on. Every element on the Websites can be further edited, formed, or shaped, by drag-and-drop arrange body of Websites or simply select an existing template.

Working in pairs is a good start for collaborative learning, in which students can learn from one another, encouraging and stimulating learning (Ministry of Science, Education and Sports, Republic Croatia, 2006). So, the main objective of this work in pairs is joint research of nanotechnology, respecting the opinions of each other and mutual supports in various areas of nanotechnology. The students design how their Webpage would look like, and start to create a Webpage, which they will continue finishing through other lessons they will learn. Presentation material, which students found of term of nano, nanotechnology, and nanomaterials, is in order to talk openly to express opinion about new topics, as well as how they see the look of their Websites, which automatically explains their organization ability within a given task.

The students explored where nanomaterials are used and in which area they are best implemented in people's lives. In addition, they research the term of "pixel," which is known to them and about photography/digital photography. Is there a connection between photography and nanotechnology? If there is any connection? Can photos be protected with nanotechnology? By intriguing questions, the students are motivated to enter deeper into the theme.

The video about "nano" and all areas of nanotechnology impress the students. The video shows that nanoparticles are tiny and invisible in their eyes. The students show interest in the entire area of nanotechnology, from what kind of material they build of and in which objects can they find them. School girls

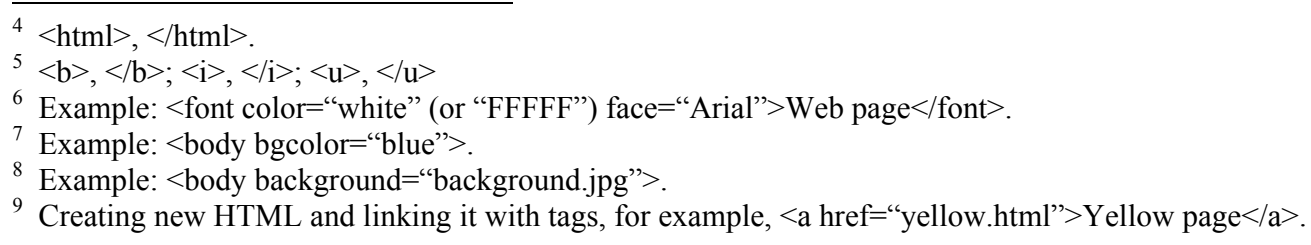


show interest in nano medicine, especially if there is any nanomaterial that exists in cosmetic, have it had any effect on their everyday life, and can it be wrong for their health and environment? School boys are interested in nanobots, especially for the fields like where they can find them and what do they look like? From all homework, the students made just one presentation about the topic, but a bunch of material of nano medicine, nano cosmetics, and nanobots. Therefore, the focus is on what the students are interested in.

\section{Lesson: The Structure of the Internet (Processing of Teaching Material and Exercise)}

The main goal of the introductory part is the students' presentation of found materials of nanotechnology, which they should (investigate) explore for homework, which shows a certain independence of students and in freely the facts if they themselves perform the task or have someone that helped.

One of the objectives is to adopt the concept of the Internet from its beginning to today, from Advanced Research Project Agency Network (ARPANET) (Computer History Museum, n.d.) to today Internet. It also aims to compare Internet connection, server, and client with nanoparticles that can send data with via nano relations in a similar way. The author explained to the students how the Internet works using the animation (see Figure 1). They compare connections between server, Internet, and client with nanoparticles that connected with nano relations and transfer data, almost the same like Internet. The author also explained server types and transfer protocols (WWW server, e-mail server, FTP server, and Domain Name Server [DNS]; Transmission Control Protocol [TCP] and Internet Protocol [IP], HTTP protocol, Simple Message Transfer Protocol [SMTP], and Post Office Protocol [POP]). The students expressed their opinions about if they have met with addresses of computers (A server has a static address, while the client has a dynamic address. With every connection to the Internet, user computers get a new address). Every computer on the Internet and in network must have its unique IP address (A computer address consists of four groups of binary numbers separate by period, which can be wrote in decimal system because of ease of memory). Symbolic addresses ${ }^{10}$ are easy to remember and comprehensible.

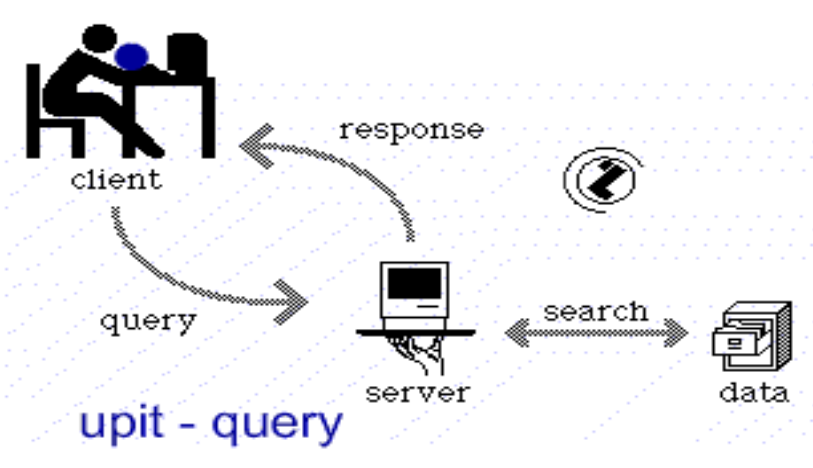

Figure 1. Server-Internet-client. ${ }^{11}$

Knowing the structure of the Internet, it is possible to describe the structure of the nanoparticles that shows a link between the sending and receiving of Internet data. With this approach, it is possible to encourage students to connect the learned words with everyday life that inspires them to logical thinking and reasoning.

\footnotetext{
${ }^{10}$ For example: http://www.os-stobrec.skole.hr.

11 http://www.znanje.org/abc/tutorials/internet_abc/01/005_client_server.htm.
} 
The students were working in pairs to research interesting facts about nanomaterials, nano medicine, nano cosmetics, nanobots, and protection of environment with nanotechnology with which they can to update their Websites. Presentation of the collected materials encourages students to independence and security in their speeches (Ministry of Science, Education and Sports, Republic Croatia, 2006) in front of other students, as well as their ability of asking if something is unclear.

As time goes by, the students are more interested in nano products, nanobots, nano medicine, nano cosmetics, and nano environmental protection. They found many YouTube videos about the topic. They are interested in whether there are some kinds of drugs that can prolong a person's life, or are there any nanoparticles in sun creams, as well as how to look like nanobots, how nanobots are made, how they affect on environment protection, etc.. Two students were sick, so the author continued working with 10 students.

\section{Lesson: Other Internet Services (Processing of Teaching Material and Exercise)}

After revision of the lessons learned in the last teaching hour about the Internet (e.g., how the Internet originates and why is it so successful, the working principle of the Internet, and explanation of types of server), the students are more clear about whether the lessons are learned or not. They explained the difference between the addresses of user computers and addresses of servers, as well as understanding of division of networks and protocols. They also shared among each other insights and interesting facts about nanotechnology.

Explanation to students of other Internet services starts from what they have known (like e-mail, Facebook, Twitter, and new one Edmodo ${ }^{12}$ ) to less known or unknown social networks, because of better understanding of the principles of operation of other social networks. The knowing advantages and disadvantages of social networks help students decide what desirable and undesirable behaviour is, as well as responsibility they bear with their actions and behaviour patterns. Creating virtual classroom on Edmodo creates greater confidence and better communication between students and teachers. In the future, it can be used to submit homework, ask questions at home, explain unclear part of material, etc. (the author's point of view). The author explained what are blog, forum, e-learning, chat, Website-hosting, and Voice Over Internet Protocol (VOIP). FTP download data directly from the link on Webpage, and mainly is used for Web-hosting Webpages on remote server. Except for Internet services, Internet offers Web tools to us. First Web tools were one-way where you can only save Webpage or read it. Today, almost every content on Internet you can share, comment, and take over on your Websites, they are interactive and called Web 2.0. ${ }^{13}$ (Toić Dlačić, Rade, Novaković, Pilipović, \& Matasić, 2014) (For those who want to learn more on tools of Web 3.0 network knowledge: It is often called "semantic Web", because in really short time, it will include intelligent browsing and looking information on what basis can decide to what group of the users belongs to the area of interest. Users will be able to submit information regarding the browsing and searching (World Wide Web Consortium [W3C], n.d.). An example of Web 3.0 is Powerset browser, so the students expressed their explanation about what Powerset browser means and would it be helpful for them in their searching for information). One of Web 2.0 is Glogster, ${ }^{14}$ which is used for creating online interactive posters. It is necessary to make an account for creating glogs. In Gloster, it is possible to create a glog where you have an opportunity to pick up custom themes, added text, pictures, audio and video, documents, link to the other pages, etc.. All these elements of glog can be edited in the similar way as we do in Weebly.

\footnotetext{
12 Social network for students and teachers.

${ }_{13}^{13}$ For example, Wordle, Glogster, and Wiki collaborative tools.

$14 \mathrm{http}: / /$ edu.glogster.com/?ref=com.
} 
Students create a glog about the interesting topic from nanotechnology (For those who want to learn more on creating mobile application on online platform IBuildApp: ${ }^{15}$ For creating a mobile application, one needs to make a registration and login in IBuildApp. First, you should create a template of mobile application, and it will show the main page of application, or just use custom templates. You can edit buttons of application, and make it that have backgrounds image or another colour of text. When you are done with creating a template, go to create an application according to the template. For creating an application, you can use the button "Change Feature" which can make changes in characteristic, for example, from HTML5 into template of offered applications (photoallergies, videos, adding social networks, and so on). When a mobile application is done, you can transfer it to a mobile phone. If somebody wants, you may start creating a mobile application about nanotechnology, nanomaterials, nanobots, nano in environmental protection, nano medicine, and nano cosmetics) (The author's point of view).

Through analysis and presentation of interactive posters created in Glogster, the students were able to see advantages and disadvantages of using various audio/video, images, and text, also better explain interesting facts about nanotechnology. Developing mobile applications encourages greater interest in solving the tasks set in front of the students. In addition, they can better demonstrate their creativity and skills, which increases their motivation and makes it persistent in performing tasks.

Some students are more interested in creating interactive posters, in general of nano medicine and nano cosmetics. Other students started turning on creating mobile applications, in general about nanobots and nanobots in nature.

\section{Lesson: Other Internet Services (Exercise and Repetition)}

For those who want to learn more about making online presentation with online software Prezi: ${ }^{16}$ Prezi is located in cloud on the Internet, which means that you have space on Internet where you can make presentation. Software is made like virtual canvas that you can zoom. On virtual canvas, you can put objects as pictures, videos, text, etc.. For start, it is important to create an account and then login in. You can choose "New Prezi" to start making a presentation. It will open a template of a possible presentation or we can choose an empty one. The title of presentation writes in box "Click to Add Title." In the box on the left side are virtual canvases like in Microsoft PowerPoint. Selecting a roll, whether insert text, while drop down menu called "Insert," allows inserting pictures, shapes, symbols, YouTube videos, background music, PDF documents, presentations, etc.. Drop down menu "Customize" allows changing themes from templates or from computer and all objects can be subsequently form (the author's point of view).

This online platform is very interesting for students because it allows making presentation of their work in an interesting and so far unknown way. Also, it arouses students' curiosity and ability to discuss and compare with the old way of making presentation.

In pair, the students choose between making online presentation-Prezi, making a mobile application-IbuildApp, or making a Website-Weebly. The topics are "nanotechnology," "nano medicine," "nano cosmetics," "nanobots," and "nanobots in nature (in environmental protection)." If you choose Weebly, it is important to connect it with interactive poster made in Glogster.

The students spontaneously began to discuss about prolonging person's life. Patients would be given fluids

\footnotetext{
${ }^{15}$ http://ibuildapp.com/.

$16 \mathrm{https}: / /$ prezi.com/.
} 
containing nano robots programmed to reconstruct the molecular structure of viruses and carcinoma. Each advocated for this problem and others against it. They asked each other questions as what happens to people if there is not enough food for prolonging life, and how would people live. Someone said that it would not be good, because it would have an effect on order in nature. The students were enthusiastic with the new software Prezi, and created a presentation on that way. They said that it is an interesting way to create a presentation, better than using Microsoft PowerPoint.

\section{Lessons: Internet Service Providers (Processing of Teaching Material and Exercise); Connect to the Internet (Processing of Teaching Material and Exercise)}

The author uses the presentation called ISP_and_Connect_to_the_Internet.pptx (On Website: Davatelji_usluga_na_Internetu_i_Povezivanje_na_Internet.pptx). The author explained to the students how the companies whose main job is to give access to the Internet name service providers or ISP. The most widely companies in Croatia are CARNet, VIP, and T-Com. CARNet is an unchargeable service provider. To connect to the Internet, you will need an open account at one of the service providers. The user account contains username, password, e-mail address and, often, a Web-hosting space for Websites. Web-hosting means setting up Website on a remote computer, which is available 24 hours, 7 days in one week. Such computers are named Web servers or WWW servers. In transferring Websites, the FTP protocol was used. There are countless examples of Web-hosting, such as http://www.free-space.net, http://www.x10hosting.com, http://www.weebly.com, etc.. The disadvantage of every free Web-hosting is that they can stop to be free or stop offer a Web-hosting. The students published Websites with the help of Weebly (steps are on presentation), which have to be checked. Toić Dlačić et al. (2014) said that before people used the analogue modems which was used a DIAL UP connection that means that the computer must adjust to call number that is given by operator and that keeps telephone line was busy. The role of the modem is to modulate data in computer for transfer through telephone wire, and later demodulate data, i.e., again adjust showing on another computer (Toić Dlačić et al., 2014). Toić Dlačić et al. (2014) described modem like digital data from computer, which turn in analogue data for transfer with telephone wire, and turn it back in digital shape on another computer. In addition, they said that Integrated Services Digital Network (ISDN) modems are a little bit faster than analogue, and are using two telephone lines. Signal was not modulate from digital to analogue, rather was transferred already in digital shape. The time spent on the Internet was measured, and thus that charged (Toić Dlačić et al., 2014). Digital Subscriber Line (DSL) has been used for all time connection with the computer, which measures the amount of data that the computer transfers with the server (Toić Dlačić et al., 2014). Asimetric Digital Subscriber Line (ADSL) has an opportunity of wireless connection with Wi-Fi card. Internet access is required to protect with password, because other devices can use traffic data. FLAT RATE is subscription with unlimited traffic data. Mobile connection, in addition to voice services and messaging, offers us data traffic (Toić Dlačić et al., 2014).

The students finish the Webpages, mobile applications, and presentations, correct the mistakes, and train display for presentation. They are enthusiastic about their Webpages and they can show their parents what they have done in class.

\section{Revision of Lesson: "Web Development" and "Internet"; Justification Achievements and Concluding Scores}

The main goal of the final part of project is to repeat everything that the students have learned during the last 12 teaching hours, how much they have developed their competence, whether they like this approach of 
teaching or not, whether students like the innovation of topics, and did they learn something new and uncommon.

The students exposed everything that they have learned about nanotechnology, terms of "nano," "nanomaterials," "nano medicine," "nano cosmetics," "nano products," "nano in environmental protection," and "nanobots." The students transfer the mobile application on their mobile phones and test how it works.

The students displayed their final works. They make three Webpages in the Website builder Weebly. With their Webpages, they were connected to YouTube videos and interactive posters, which are made in Glogster, also they were able to link other pages on the Internet, and all materials that were interesting to them about nanotechnology. In addition, they made one presentation in Prezi, which talked, in general, about nanotechnology and nanomaterials. In the end, they made two mobile applications in IBuildApp.

Final Works of Students in 7 th Grade $^{17}$

Petra Cokarić i Katarina Kolovrat: Weebly \& Glogster (http://nanomedicina.weebly.com/);

Katarina Garac: Weebly \& Glogster (http://nanoproizvodi.weebly.com/);

Paulina Grčić i Magdalena Brekalo: Weebly \& Glogster (http://nanokozmetika.weebly.com/);

Marijo Gajšak i Stipe Bilonić: IBuildApp-Aplikacija_o_nanobotima.docx;

Marino Kovačić: IBuildApp-Aplikacija_o_nanobotima_u_prirodi.docx;

Antonela Sičenica i Barbara Blažević: Prezi-nanotehnologija-5pja82ibubtb (zipped folder).

\section{Conclusion}

In daily classes, it is necessary to introduce innovations, in which students gain new experience and competences and where they have an opportunity of collaborative working. Mutual learning among the students results in discovering affection in various fields of informatics, which encourages them to respect each other's opinions and create personal critical thinking. In addition, students are able to connect nanotechnology with various areas of Science, Technology, Engineering, and Mathematics (STEM) subjects, as well as they could improve their English language. Introducing the new online platforms, such as Weebly, IBuildApp, Glogster, or Prezi, the students have become interested in the completion of its existing knowledge by exploring new opportunities for realization of tasks and interest of knowing what is behind each section of, for example, Websites, Internet, or mobile application. That allows students to make logical and scientific connection of facts learned from whole lesson plans "Web development" and "the Internet," as well as connecting with the facts of everyday life. The students' curiosity of prolonging people's life opens a very interesting discussion, which helps them make their opinion of this burning topic.

This work has helped to see how the students think about new terms, those that they have heard a little and do not have a certain opinion. Although with innovative methods of teaching and learning, there is more conscientious students' willingness and motivation to complete tasks and better learning and understanding of teaching materials.

\section{References}

Computer History Museum. (n.d.). Internet history 1962 to 1992. Retrieved from http://www.computerhistory.org/internethistory/ Czwyk Marić, A. (2014a). Digiskills: Nanotechnology and nanocomputers-The original proposal of good practice. Retrieved from http://www.digiskills-project.eu/?q=content/nanotechnology-and-nanocomputers

${ }^{17}$ You may find all lesson plans, presentations, and student work on the author's Webpage http://7thgradenano.weebly.com/. 
Czwyk Marić, A. (2014b). Nanotechnology. Retrieved from http://nanotechnologyhr.weebly.com/

Mid-continet Research for Education and Learning (McREL). (2009). Delivering and practical guidance to educators. Retrieved from http://www.mcrel.org/NanoTeach/project-goals/nanoscience-technology.asp

Ministry of Science, Education and Sports, Republic Croatia. (2006). Curriculum for primary school. Zagreb: Ministry of Science, Education and Sports.

National Nanotechnology Initiative. (n.d.). What is nanotechnology? Retrieved from http://www.nano.gov/nanotech-101/ what/definition

Toić Dlačić, K., Rade, D., Novaković, L., Pilipović, V., \& Matasić, I. (2014). Like it 7, textbook in informatics for 7th grade of primary school, Zagreb: Alfa.

UnderstandingNano.com. (n.d.). Nanoparticle applications and uses. Retrieved from http://www.understandingnano.com/ nanoparticles.html

World Wide Web Consortium (W3C). (n.d.). Semantic Web. Retrieved from http://www.w3.org/standards/semanticWeb/ 